\title{
The mechanism of mammalian mitochondrial quality control system
}

\section{Safir Ullah Khan ${ }^{1,}{ }^{*}$, Munir Ullah Khan ${ }^{2}$}

1Hefei National Laboratory for Physical Sciences at the Microscale, School of Life Sciences, University of Science and Technology of China, Hefei 230027, China.

${ }^{2}$ MOE Key Laboratory of Macromolecular Synthesis and Functionalization, Department of Polymer Science and Engineering, Zhejiang University, Hangzhou 310027, China.

\begin{tabular}{lllllll}
\hline Received: 04.11.2021 & $\bullet$ & Accepted: 15.12.2021 & $\bullet$ & Published: 31.12.2021 & $\bullet$ & Final Version: 31.12.2021 \\
\hline
\end{tabular}

\begin{abstract}
The mitochondria play an essential role in cellular metabolism by acting as powerhouses. It is also involved in several biological processes such as cell metabolism, stress signaling, calcium homeostasis, reactive oxygen species (ROS) and apoptosis. Maintaining cellular physiological function is strongly dependent on mitochondrial quality control. Mitochondrial malfunction will lead to various disorders. For this review, we evaluate the current understanding of the molecular mechanism of mitochondria quality control, which might also serve as an asset for organisms' health and the prevention of disease.
\end{abstract}

Keywords: mammals; mitochondrial dynamics; mitochondrial Autophagy; protein quality control

\section{Introduction}

Mitochondria are important organelles in eukaryotic cells. Peroxy phosphorylation provides energy for cell life activity. It is involved in Cellular metabolism and stress signaling, calcium homeostasis, reactive oxygen species (reactive oxygen species, ROS) production, and cell regulation apoptosis Process. Many studies have shown that the Regulation of mitochondrial homeostasis is disturbed or the dysfunction is related to human Parkinson's disease, Alzheimer's disease, and diabetes [1-3], which can alter oocytes in animals, resulting in reproductive dysfunction [4]. Therefore, maintaining mitochondria's normal quality and function in animal cells is essential. It plays an important role in health and disease reduction.

At present, there are many pathways in animal cells to regulate mitochondria to prevent mitochondrial dysfunction. For example, mitochondria antioxidant system, Alleviating the Toxic Effect of ROS by Chemical System [5], within the mitochondrial matrix Chaperones, Proteases and Ubiquitin Proteasome Systems (UPS) Regulates Mitochondrial Protein Expression [6]. Mitochondria change shape by fusion and division, making the lines Intergranular content to communicate or separate damaged mitochondria through Autophagy. In this review paper, we mainly focused on mammalian mitochondria, their molecular mechanism of homeostasis regulation, mitochondrial reactive oxygen species, Autophagy, Protein Quality Control, and Mittinderived Vesicles in Mitochondrial Quality maintain homeostasis by regulating mitochondrial quality[7].

\footnotetext{
* Corresponding Author: author@institute.xxx
} 


\subsection{Mitochondrial quality control of reactive oxygen species}

Reactive oxygen species ROS is a class of chemical substances containing reactive oxygen atoms, including ultra Oxygen ions, hydroxyl radicals, hydrogen peroxide. Currently, it is believed that ROS in animal cells is mainly composed of mitochondrial respiratory chain complex I and III[8], and its excessive production will oxidize and destroy mitochondria DNA mtDNA.The structure and function of essential molecules such as plasma membrane and respiratory chain complex lead to Cause cells being in a state of oxidative stress [9]. To prevent the excessive accumulation of ROS from oxidizing mitochondria, the antioxidant enzyme system in animal cells will be responsible for removing too much ROS to maintain the redox state's stability. Mitochondria Antioxidant enzyme system reduces ROS to cause oxidative stress on animal cells; at the same time, a low level of ROS is maintained, making it a signal molecule Participate in mitochondrial quality control regulation, activate the cellular antioxidant system, This prevents ROS-induced oxidative stress from affecting mitochondrial DNA and protein Qualitative destruction[5, 10].

\subsection{Mitochondrial quality control at the organelle level}

\subsubsection{The Regulation of mitochondrial homeostasis on mitochondrial quality control.}

Mammalian cell mitochondria are highly motile State organelles that frequently fuse and divide. Its quality is an important way of controlling mitochondrial morphology and function [11]. The fusion between mitochondria of mammalian cells depends on an A series of mitochondrial transmembrane GTPases, mainly including localization outside the mitochondria Membrane fusion protein 1/2 (mitofusin 1/2, MFN 1/2) and inner membrane Optic atrophy 1, OPA1, of which MFN $1 / 2$ mitochondrial outer membrane fusion. Studies have found that these regulatory mitochondrial fusion protein genes (Especially MFN 1/2), after inhibition of mitochondria, not Only its morphology is fragmented, but also blocking the interaction of the content between mitochondria, and reduce the mtDNA content and membrane potential, resulting in mitochondria Decreased oxidative Phosphorylation [12, 13]. Another study found that MFN 2-dimensional Maintained the level of Coenzyme Q in the electron transport chain [14], OPA1 maintained the morphology of the mitochondrial cristae promotes the assembly of the respiratory chain complex [15]. The above research results suggest that the mitochondrial fusion factor promotes mitochondria Fusion and affects morphological changes while enhancing mitochondrial oxidation. MFN 1/2 and other proteins involved in mitochondrial outer membrane fusion, the molecular mechanism of coregulation is poorly understood. It is worth noting that MFN ubiquitination is proposed to be essential for regulating mitochondrial outer membrane. The degradation of the proteasome during stress is related to the inhibition of fusion. For example, the study found that the E3-ubiquitin ligase Parkin relied on PTEN to induce putative kinase 1(PINK1), facilitating depolarized mitochondrial MFN 1/2 induces an essential process before mitochondrial Autophagy[16]. Recent research shows that the non-degradation of MFN after Ubiquitination promotes the mitochondrial fusion of animal cells[17]. However, currently, about E3 ubiquitin-protein ligase makes MFN ubiquitin. The mechanism of non-degradation after chemical conversion is still unclear and needs further study. OPA1 Is an essential protein that regulates the fusion of the inner mitochondrial membrane. The influence of fusion depends on transcription and post-translational Regulation. For example, It is believed that OPA1 is involved in YME1-like 1 ATPase. The formation of long and short oligomers promotes the fusion of the inner mitochondrial membrane [18]. However, under stress conditions, Induced mitochondrial inner membrane protease OMA1 (overlapping with themAAA protease 1) completely degrade long OPA1, promoting the mitochondrial division [19]. 
Mitochondrial division is mainly affected by triphosphate in the cytoplasm Glycoside (guanosine triphosphate, GTP) enzyme, generally recognized for the mitochondrial division, the endoplasmic reticulum and mitochondria form a structural couple, where (mitochondria-associated E.R. membrane, MAM), linked them to contract locally, and at the same time recruiting DRP1 and Receptor fission protein 1 on the outer mitochondrial membrane 1, FIS1, mitochondria fission factor (mitochondria fission factor, MFF) and mitochondrial dynein 49/51 (mitochondrial dynein proteins of 49 and 51, MID49/51) assemble into oligomers, hydrolyze GTP to split the mitochondrial membrane [20]. The close study found that FIS1 can assist DRP1 in promoting mitochondrial division. The role of the surface is smaller than that of MFF and MID49/51 [21]. Another study found that MFF and MID coexist at the same mitochondrial division site. It shows that these proteins may synergistically promote DRP1-mediated mitochondria Split [22]. However, the proteins such as DRP1, MFF, and MID, how the coordination regulates mitochondrial division are still unclear and need in-depth study.

It is generally believed that mitochondrial division is closely related to cell apoptosis. DRP1 can promote mitochondrial division and mitochondrial cristae morphology, leading to the release of cytochrome $\mathrm{c}$ and inducing apoptosis. However, recent studies have shown that DRP1-dependent mitochondrial division remodels the cristae of the mitochondria with the assistance of MID49/51, which promotes cell Apoptosis, indicating that these split proteins regulate the mitochondria by inducing apoptosis [23].

Some studies have suggested that the recruitment of DRP1 to the outer mitochondrial membrane is dependent on Post-transcriptional modifications, mainly including DRP1 phosphate, glycosylation, Ubiquitination, sulfhydryl, and nitrosylation [24]. Stress stimulates the mitochondria, causing them to activate kinases, Phosphorylate serine residues on the DRP1 protein. For example, protein kinase A Phosphorylated Serine 637 of DRP1 inhibits mitochondria division; On the contrary, the cell cycle regulatory protein (CDK1-cyclin B) makes DRP1 Phosphorylation of amino acid 616 promotes cell division $[25,26]$.

The recruitment and activation of DRP1 are also subject to the Regulation of mitochondrial outer membrane receptor proteins. Therefore, future research should Clarify the DRP1 protein posttranslational modification and the mitochondrial outer membrane receptor on how proteins cooperate to regulate the recruitment and activation of DRP1.

In addition, animal cells are stimulated by internal and external signals and different In the case of energy demand, mitochondria regulate through fusion and division, control their shape and distribution, and increase their number and distribution through biosynthesis. The copy number of mtDNA promotes the protein related to the respiratory chain complex Gene expression to improve oxidative metabolism, which is essential for maintaining and repairing the standard structure and function of mitochondria. The current research has proved that many signal molecules inside and outside the cell regulate mitochondrial biosynthesis. Among them, peroxisome proliferatoractivated receptor $\gamma$ co-activates Factor-1 $\alpha$ (peroxisome-proliferator-activated receptor $\gamma$ coactivator- $1 \alpha$, PGC- $1 \alpha$ ) is considered to regulate mitochondrial growth. Transcriptional coactivator plays a central role in biosynthesis, mainly related to downstream nuclear respiratory factor 1/2 (NRF1/2). Gene binding activates transcription. The increase in NRF1/2 levels promotes mitochondrial proteins and mitochondrial transcription factors encoded by the nuclear genome Sub-A (mitochondrial transcription factor A, TFAM) gene Express. After TFAM enters the mitochondria, it promotes the development of mtDNA coding genes Expression and then regulates the biosynthesis of mitochondria [27]. 
The current research believes that various protein factors regulate the quality of mtDNA. For example, The oligomeric cyclic ATP-dependent protein degrades TFAM, Maintains the stability of TFAM/mtDNA, and regulates mtDNA And Expression [28]. PARKIN is an E3-ubiquitin ligase, which mainly participates in Autophagy reaction with mitochondria. The study found that PARKIN can interact with TFAM binds to promote mitochondrial biosynthesis. When ROS accumulates too much within mitochondria, PARKIN can also bind to mtDNA and maintain the stability of mtDNA $[29,30]$.

\subsubsection{Autophagy regulates mitochondrial quality control.}

Mitophagy refers to the passage of cells through the autophagy mechanism. The selective removal of damaged mitochondria is essential for maintaining mitochondria homeostasis. Cytochrome c and calcium ions induce apoptosis, and mitochondria pass through Phagocytosis, remove abnormal mitochondria, and prevent healthy mitochondria Fusion with damaged mitochondria, thereby inhibiting the stimulation of apoptosis pathways and maintaining the survival of cells. The current research suggests that the degeneration of mitochondria Polarization may induce mitochondrial Autophagy, Autophagyuter mitochondrial membrane stimulates Enzymes PINK1, and E3-ubiquitin ligase PARKIN involved in the potential membrane [31, 32]. PARKIN is An E3-ubiquitin ligase, mainly located in the cytoplasm, enough to recruit PARKIN to target damaged mitochondria. Under physiological conditions, PINK1 is transported to the inner mitochondrial membrane, linked with presenilin-associated rhomboid protein (PARL), and processing peptidase (mitochondrial processing peptidase, MPP) degrades. However, after mitochondrial depolarization, PARL and MPP protease activity decreased, But the specific molecular mechanism is still unclear [33]. After PARL and MPP are inactivated, they inhibit PINK1 from transferring to the inner mitochondrial membrane, which causes it to be located in the outer mitochondrial membrane with translocases in the outer membrane (translocase of the outer mitochondrial membrane (TOM). Subsequently, the complex is connected with PINK1 phosphorylates PARKIN, increasing its ubiquitin ligase activity, resulting in some mitochondria Ubiquitination of outer membrane and cytoplasmic proteins, initiation autophagy mechanism, resulting in the degradation of damaged mitochondria [16, 34, 35].

PARKIN can act on a variety of target proteins, including MFN 1/2, TOM complex member, apoptotic protein, members of the autophagy mechanism. It mediates these proteins through multiple Induction of mitochondrial Autophagy in various ways, For example, induce MFN 1/2, the mitochondrial Rho-GTPase and other proteins involved in mitochondrial fusion and transport via proteases Body degradation, inhibit targeted damage to mitochondria and standard mitochondria Body fusion, leading to its degradation, promoting p62 and NBR1 (neighbor of BRCA1 gene 1) and other autophagy adaptor proteins.

These have ubiquitin junction region and microtubule-associated protein one light chain of these three proteins, The interaction area can anchor the autophagosome membrane near the targeted mitochondria, Accelerate the formation of autophagosomes and break down damaged mitochondria [36, 37], or stimulate Live E3 ubiquitin ligase and Beclin-1 regulate autophagy proteins 1, Start mitochondrial Autophagy [Autophagyver, currently about PARKIN-mediated mechanism of mitochondrial Autophagy target protein ubiquitination is not well understood. Indeed, further investigation is still needed[38].

Many studies have shown that the outer mitochondrial membrane of animal cells consists of BNIP3(Bcl-2 and adenovirus E1B19 kDa-interacting protein3) and NIX (NIP3-like protein X) and 
other autophagy receptor proteins, inducing not only apoptosis but also mitochondrial Autophagy $[39,40]$. BNIP3 and NIX are the Bcl-2 families that Contain only Bcl-2 homology domain 3 (Bcl-2 homology 3, BH3) are members of the subfamily, and their protein has 56\% sequence Homology. The N-terminus contains a consistent light chain of microtubule-associated protein 3(microtubuleassociated protein light chain 3, LC3) Interaction region, which can directly connect to LC3 protein and recruit autophagosomes[41]. Degrade mitochondria through a method independent of the PINK1/PARKIN system Type regulation of mitochondrial Autophagy. In addition, the study found that NIX can compete with PARKIN interacts to promote PARKIN to recruit depolarized mitochondria Body [42]. The latest research found that BNIP3 can interact with PINK1 and inhibit PINK1 is lysed to promote its accumulation in the outer mitochondrial membrane while recruiting PARKIN, enhances mitochondrial Autophagy latest findings suggest that BNIP3/NIX not only has the function of directly inducing mitochondrial Autophagy, Autophagy also promote mitochondria by adjusting the PINK1/PARKIN system through Autophagy[43].

\subsection{Autophagy mitochondrial quality control at protein level}

\subsubsection{The Regulation of mitochondrial proteases on mitochondrial quality.}

In animal cells, most nuclear genes encode for mitochondrial proteins. These proteins pass through a highly selective mechanism transported to the mitochondria [44]. Mitochondrial outer and inner membrane translocation enzymes chaperone and protease act synergistically to promote protein migration, the cleavage and folding of the targeting sequence is in its original form. Mitochondrial heat shock protein 60 (HSP60) directly transports and folds these proteins and regulates these transporters [45]. HSP70 is another type within the mitochondrial matrix involved in proteins' transport, folding, and assembly. It also promotes the formation of iron-sulfur clusters [46]. Mitochondrial HSP60 and HSP70 play a role in protein folding and electron transport chain assembly. However, they also cooperate to prevent unfolding and misfolding Protein aggregation [47].

Unfolded and assembled proteins in the mitochondrial membrane space and matrix are degraded by a series of proteases. The mitochondrial matrix mainly contains Three AAA proteases involved in protein degradation, including Lon and casein. Protease $\mathrm{P}$ (caseinolytic protease $\mathrm{P}, \mathrm{ClpP}$ ) are two soluble Protease and a membrane-bound m-AAA protease of which Lon Preference to degrade oxidized, and misfolded proteins, mitochondrial proteins under cytotoxic stress, ClpP is activated to initiate mitochondrial protein non-folding Fold reaction and degrade the misfolded protein[48, 49]. The m-AAA protein Enzymes are oligomeric proteases with multiple functions and assembly of oxidative phosphorylation complex subunits acting similar to the function of the molecular chaperone [50]. The activity of these enzymes is to regulate the mitochondrial ribosomal protein MRPL32 and the function of the fusion protein OPA1 [51, 52].

The protein quality of the mitochondrial membrane space is affected by the membrane-bound iAAA protein Enzyme and soluble protease HtrA2/Omi control. i-AAA protease Involved in maintaining the processing of oxidative phosphorylation complex and OPA1. Research shows that mammalian cells stimulated by apoptosis signals, HtrA2/Omi Release into the cytoplasm, c-IAP1 (c-inhibitor of apoptosis 1), XIAP(X-linked inhibitor of apoptosis) Wait for degradation, and then initiate cell apoptosis [53]. Non-apoptotic Under the file, HtrA2(high-temperature requirement A2)/Omi bit In the mitochondrial intermembrane cavity, it interacts with proteins involved in mitochondrial fusion and Autophagy[54]. 
The inner mitochondrial membrane contains two proteases in mitochondrial quality control: Metallopeptidase OMA1 and membrane-bound i-AAA protein. The enzyme functions similarly as a stress protease by processing OPA1. Determine the morphology of mitochondria. The rhomboid protease PARL may also be involved in OPA1 processes and cleaves the autophagy protein PINK1, preventing healthy mitochondrial Autophagy[55].

\subsubsection{Regulation of mitochondrial quality by the ubiquitin-proteasome system.}

Ubiquitin proteasome system UPS is the key to selective protein degradation in mammalian cytoplasm. To be systematic, the process of degrading protein is complicated after the action of ubiquitin-conjugating enzyme E2 and ubiquitin ligase E3 and passes through the ubiquitinactivating enzyme E1 ubiquitin. Moreover, the outer membrane protein faces the cytoplasm and is most likely to be reduced by UPS ubiquitination Solution [56]. In addition, the protein in the matrix can also be transported to the mitochondria. It can be degraded after being labeled by UPS in the cytoplasm [57]. Due to mitochondrial membrane, The compartment, inner membrane, and matrix are separated from the cytoplasm, and the UPS system lacks. There is experimental evidence in mitochondria's inner membrane and matrix, so people first believe that the proteins located in these regions of the mitochondria cannot be. The UPS system in the cytoplasm is regulated and can only be reduced by specific proteases Solutions [58]. However, current studies have found that the mitochondrial intermembranous cavity, intima, and Matrix proteins can also be modified by Ubiquitination[59, 60], these mitochondria The body area contains the USP system, and it is speculated that the translation or folding of the wrong egg Before white matter reaches the characteristic site of mitochondria, it will be present in the mitochondria. The USP system is degraded. Studies have found that oxidized phosphoric acid in mitochondria The matrix proteins of the subunits of the chemical complex may also be reversely transported into the cytoplasm degraded by UPS [57]. Therefore, the current research has fully established the UPS regulation of cell mitochondrial function, and it may be through a variety of ways to Mitochondria implement quality control.

\subsubsection{The Regulation of mitochondrial quality by mitochondrial-derived vesicles.}

Mammalian cell mitochondria will derive packages after being stressed. Vesicles containing proteins from the outer mitochondrial membrane, inner membrane, and matrix are then transported to the lysosome or peroxisome to regulate the Granular line mass. At present, the vesicles formed after mitochondrial stress are still unclear how it is transported to peroxisomes, but research suggests that transport. The vesicles transported to the lysosome are rich in oxidized proteins, and are Enzymes are degraded in vivo[61].

It is worth noting that the study found that the PINK1 and PARKIN series system not only regulates mitochondrial Autophagy bAutophagyarticipates in mitochondrial oxygen Vesicle formation induced by chemical stress, which is related to autophagy protein 5[62]. Based on the current research results, scholars speculate that mitochondria A large amount of ROS produced by body oxidative phosphorylation may oxidize matrix proteins or make Protein assembly errors, causing the aggregation of these proteins. At the same time, Phospholipids are oxidized to produce phosphatidic acid, which changes the curvature of the membrane and protein. The mass aggregates can saturate the molecular chaperones, resulting in protein Failure to locate and transport via specific channels. Cardiolipin is oxidized. Later, it will further interfere with the transport channel [63]. Subsequently, PINK1 was quickly transported and gathered on the channel that had lost its 
transport function. PINK Phosphorus Acidifies the ubiquitin ligase region of PARKIN to stably recruit activated PARKIN. PARKIN makes some proteins located on the outer mitochondrial membrane. After Ubiquitination, it causes the mitochondrial outer membrane to reshape, bend, and become visible publicly [64].

However, when the mitochondria have been depolarized or malfunctioning, the compound PINK is deposited in all of the mitochondria's transport channels. Recruit PARKIN to ubiquitinate various proteins in the outer mitochondrial membrane, activate the autophagy mechanism, phagocytize the entire mitochondria [65]. These findings suggest that mitochondria form vesicles to remove damaged proteins - a vital mechanism to maintain mitochondrial quality and prevent dysfunction.

\section{Conclusion and discussion}

In summary, mammalian cells have various mitochondria control pathways, mainly regulated from protein and organelle levels. Mitochondrial quality improves mitochondrial function to ensure the health of the body (Figure 1). The classic idea is that animal cells have a different mitochondrial regulatory network. The quantity control system is usually a highly layered regulation network. However, significant progress has been made in the mitochondrial quality control system. It has been established that these quality control systems are not stratified Regulatory network, but through cross-action Regulation of mitochondrial quality, and The occurrence of a series of related events depends on stress stimulus, activation of cofactor And mitochondrial damage, but these quality control systems It is not clear how to transform and identify mitochondrial stress signals. Indeed, further studies are needed.

In addition, the mitochondrial quality control system in different compartments of mitochondria is not very clear on how to coordinately regulate mitochondrial function. These regulatory factors can activate or inhibit different mitochondrial quality control. Access is also uncertain and will require further investigation in the future by understanding the lines of a granulocyte quality control system that can screen for ways to increase mitochondrial quality in order to develop a therapy for diseases caused by mitochondrial dysfunction.

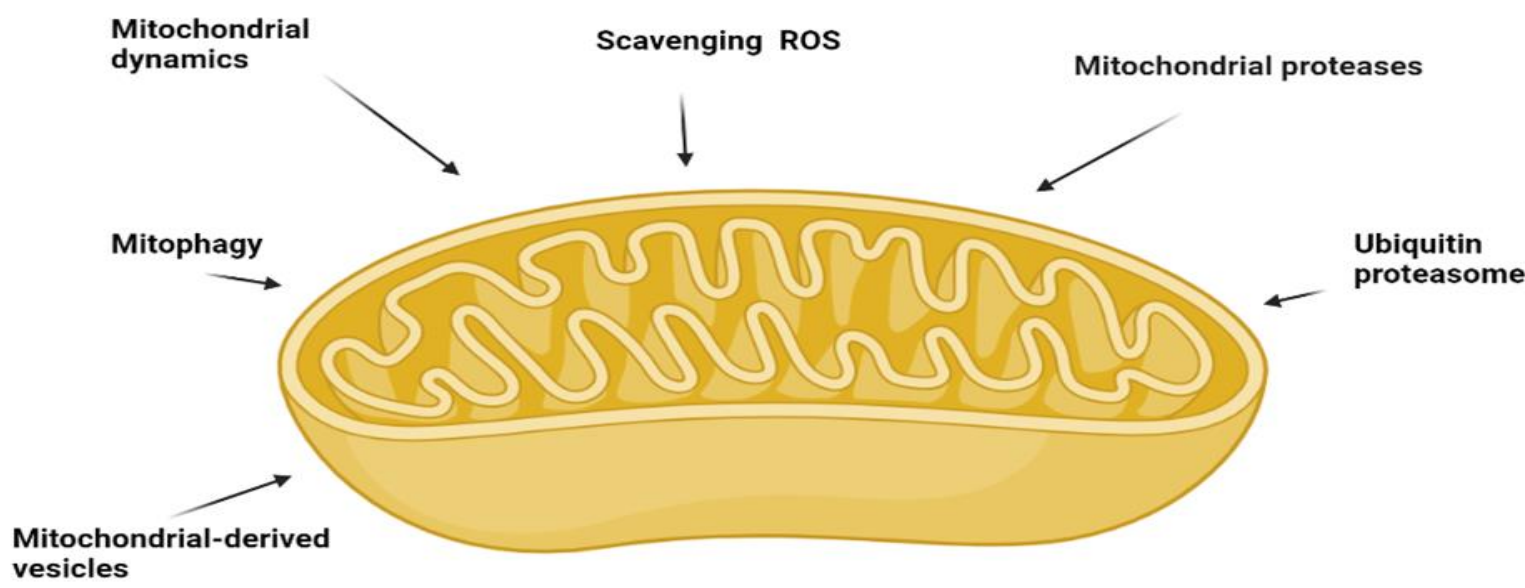

Mitochondrial quality control

Figure 1. Mammalian cell mitochondria quality control mechanisms 
Antioxidant defense systems in mammalian cells act to detoxify excess ROS to help protect the mitochondria from its damaging effects. Mitochondrial dynamics are required to maintain mitochondrial shape, number, and mtDNA stability. Mitophagy allows mammalian cells to remove dysfunctional mitochondria, maintaining a healthy mitochondria network selectively. To control mitochondrial activity, proteases and ubiquitin-proteasome systems degrade mitochondrial protein components.

\section{Author contribution}

Safir Ullah Khan devised and designed the study. Munir Ullah Khan performed survey and analysis data. The author read and approved the manuscript.

\section{Declarations}

Conflict of interest The authors declare no potential conflicts of interest.

Ethical approval This article does not contain any studies with human participants or animals performed by the author.

Informed consent No informed consent is needed for this article.

\section{References}

[1] Siddiqui, A., Mitochondrial quality control via the PGC1 $\alpha$-TFEB signaling pathway is compromised by parkin Q311X mutation but independently restored by rapamycin. Journal of Neuroscience, 2015. 35(37): p. 12833-12844.

[2] Yin, F., Mitochondrial function in ageing: coordination with signalling and transcriptional pathways. The Journal of physiology, 2016. 594(8): p. 2025-2042.

[3] Zamora, M., R. Pardo, and J.A. Villena, Pharmacological induction of mitochondrial biogenesis as a therapeutic strategy for the treatment of type 2 diabetes. Biochemical pharmacology, 2015. 98(1): p. 1628.

[4] Schatten, H., Q.-Y. Sun, and R. Prather, The impact of mitochondrial function/dysfunction on IVF and new treatment possibilities for infertility. Reproductive Biology and Endocrinology, 2014. 12(1): p. 1-11.

[5] Zorov, D.B., M. Juhaszova, and S.J. Sollott, Mitochondrial reactive oxygen species (ROS) and ROSinduced ROS release. Physiological reviews, 2014. 94(3): p. 909-950.

[6] Kotiadis, V.N., M.R. Duchen, and L.D. Osellame, Mitochondrial quality control and communications with the nucleus are important in maintaining mitochondrial function and cell health. Biochimica et Biophysica Acta (BBA)-General Subjects, 2014. 1840(4): p. 1254-1265.

[7] Youle, R.J. and A.M. Van Der Bliek, Mitochondrial fission, fusion, and stress. Science, 2012. 337(6098): p. 1062-1065.

[8] Brand, M.D., The sites and topology of mitochondrial superoxide production. Experimental gerontology, 2010. 45(7-8): p. 466-472.

[9] Elfawy, H.A. and B. Das, Crosstalk between mitochondrial dysfunction, oxidative stress, and age related neurodegenerative disease: Etiologies and therapeutic strategies. Life sciences, 2019. 218: p. 165-184.

[10] Murphy, M.P. and R.C. Hartley, Mitochondria as a therapeutic target for common pathologies. Nature Reviews Drug Discovery, 2018. 17(12): p. 865-886.

[11] Horbay, R. and R. Bilyy, Mitochondrial dynamics during cell cycling. Apoptosis, 2016. 21(12): p. $1327-$ 1335.

[12] Chen, H., A. Chomyn, and D.C. Chan, Disruption of fusion results in mitochondrial heterogeneity and dysfunction. Journal of Biological Chemistry, 2005. 280(28): p. 26185-26192. 
[13] Olichon, A., Loss of OPA1 perturbates the mitochondrial inner membrane structure and integrity, leading to cytochrome c release and apoptosis. Journal of Biological Chemistry, 2003. 278(10): p. 77437746.

[14] Mourier, A., Mitofusin 2 is required to maintain mitochondrial coenzyme Q levels. Journal of Cell Biology, 2015. 208(4): p. 429-442.

[15] Alavi, M.V. and N. Fuhrmann, Dominant optic atrophy, OPA1, and mitochondrial quality control: understanding mitochondrial network dynamics. Molecular neurodegeneration, 2013. 8(1): p. 1-12.

[16] Bernardini, J., M. Lazarou, and G. Dewson, Parkin and mitophagy in cancer. Oncogene, 2017. 36(10): p. 1315-1327.

[17] Yue, W., A small natural molecule promotes mitochondrial fusion through inhibition of the deubiquitinase USP30. Cell research, 2014. 24(4): p. 482-496.

[18] Song, Z., OPA1 processing controls mitochondrial fusion and is regulated by mRNA splicing, membrane potential, and Yme1L. Journal of Cell Biology, 2007. 178(5): p. 749-755.

[19] Baker, M.J., Stress - induced OMA 1 activation and autocatalytic turnover regulate OPA 1 - dependent mitochondrial dynamics. The EMBO journal, 2014. 33(6): p. 578-593.

[20] Otera, H., Mff is an essential factor for mitochondrial recruitment of Drp1 during mitochondrial fission in mammalian cells. Journal of Cell Biology, 2010. 191(6): p. 1141-1158.

[21] Palmer, C.S., MiD49 and MiD51, new components of the mitochondrial fission machinery. EMBO reports, 2011. 12(6): p. 565-573.

[22] Elgass, K.D., Analysis of ER-mitochondria contacts using correlative fluorescence microscopy and soft X-ray tomography of mammalian cells. Journal of cell science, 2015. 128(15): p. 2795-2804.

[23] Otera, H., Drp1-dependent mitochondrial fission via MiD49/51 is essential for apoptotic cristae remodeling. Journal of Cell Biology, 2016. 212(5): p. 531-544.

[24] Otera, H., N. Ishihara, and K. Mihara, New insights into the function and regulation of mitochondrial fission. Biochimica et Biophysica Acta (BBA)-Molecular Cell Research, 2013. 1833(5): p. 1256-1268.

[25] Cribbs, J.T. and S. Strack, Reversible phosphorylation of Drp1 by cyclic AMP - dependent protein kinase and calcineurin regulates mitochondrial fission and cell death. EMBO reports, 2007. 8(10): p. 939-944.

[26] Taguchi, N., Mitotic phosphorylation of dynamin-related GTPase Drp1 participates in mitochondrial fission. Journal of Biological Chemistry, 2007. 282(15): p. 11521-11529.

[27] Villena, J.A., New insights into PGC - 1 coactivators: redefining their role in the regulation of mitochondrial function and beyond. The FEBS journal, 2015. 282(4): p. 647-672.

[28] Matsushima, Y., Y.-i. Goto, and L.S. Kaguni, Mitochondrial Lon protease regulates mitochondrial DNA copy number and transcription by selective degradation of mitochondrial transcription factor A (TFAM). Proceedings of the National Academy of Sciences, 2010. 107(43): p. 18410-18415.

[29] Kuroda, Y., Parkin enhances mitochondrial biogenesis in proliferating cells. Human molecular genetics, 2006. 15(6): p. 883-895.

[30] Rothfuss, O., Parkin protects mitochondrial genome integrity and supports mitochondrial DNA repair. Human molecular genetics, 2009. 18(20): p. 3832-3850.

[31] Narendra, D., Parkin is recruited selectively to impaired mitochondria and promotes their autophagy. Journal of cell biology, 2008. 183(5): p. 795-803.

[32] Narendra, D.P., PINK1 is selectively stabilized on impaired mitochondria to activate Parkin. PLoS biology, 2010. 8(1): p. e1000298.

[33] Stotland, A. and R.A. Gottlieb, Mitochondrial quality control: easy come, easy go. Biochimica et Biophysica Acta (BBA)-Molecular Cell Research, 2015. 1853(10): p. 2802-2811.

[34] Greene, A.W., Mitochondrial processing peptidase regulates PINK1 processing, import and Parkin recruitment. EMBO reports, 2012. 13(4): p. 378-385.

[35] Lazarou, M., Role of PINK1 binding to the TOM complex and alternate intracellular membranes in recruitment and activation of the E3 ligase Parkin. Developmental cell, 2012. 22(2): p. 320-333.

[36] Kirkin, V., A role for NBR1 in autophagosomal degradation of ubiquitinated substrates. Molecular cell, 2009. 33(4): p. 505-516.

[37] Pankiv, S., p62/SQSTM1 binds directly to Atg8/LC3 to facilitate degradation of ubiquitinated protein aggregates by autophagy. Journal of biological chemistry, 2007. 282(33): p. 24131-24145.

[38] Orvedahl, A., Image-based genome-wide siRNA screen identifies selective autophagy factors. Nature, 2011. 480(7375): p. 113-117. 
[39] Chakrama, F.Z., GABARAPL1 (GEC1) associates with autophagic vesicles. Autophagy, 2010. 6(4): p. 495-505.

[40] Lee, Y., Mitochondrial autophagy by Bnip3 involves Drp1-mediated mitochondrial fission and recruitment of Parkin in cardiac myocytes. American journal of physiology-heart and circulatory physiology, 2011. 301(5): p. H1924-H1931.

[41] Gao, A., Bnip3 in mitophagy: Novel insights and potential therapeutic target for diseases of secondary mitochondrial dysfunction. Clinica Chimica Acta, 2020. 506: p. 72-83.

[42] Hamacher-Brady, A. and N.R. Brady, Mitophagy programs: mechanisms and physiological implications of mitochondrial targeting by autophagy. Cellular and molecular life sciences, 2016. 73(4): p. 775-795.

[43] Ma, P., Atg1-mediated autophagy suppresses tissue degeneration in pink1/parkin mutants by promoting mitochondrial fission in Drosophila. Molecular biology of the cell, 2018. 29(26): p. 3082-3092.

[44] Zhang, T., BNIP3 protein suppresses PINK1 kinase proteolytic cleavage to promote mitophagy. Journal of biological chemistry, 2016. 291(41): p. 21616-21629.

[45] Chakraborty, K., Chaperonin-catalyzed rescue of kinetically trapped states in protein folding. Cell, 2010. 142(1): p. 112-122.

[46] Dutkiewicz, R., Ssq1, a mitochondrial Hsp70 involved in iron-sulfur (Fe/S) center biogenesis: similarities to and differences from its bacterial counterpart. Journal of Biological Chemistry, 2003. 278(32): p. 29719-29727.

[47] Pellegrino, M.W., A.M. Nargund, and C.M. Haynes, Signaling the mitochondrial unfolded protein response. Biochimica et Biophysica Acta (BBA)-Molecular Cell Research, 2013. 1833(2): p. 410-416.

[48] Koppen, M. and T. Langer, Protein degradation within mitochondria: versatile activities of AAA proteases and other peptidases. Critical reviews in biochemistry and molecular biology, 2007. 42(3): p. 221-242.

[49] Haynes, C.M., ClpP mediates activation of a mitochondrial unfolded protein response in C. elegans. Developmental cell, 2007. 13(4): p. 467-480.

[50] Voos, W., Chaperone-protease networks in mitochondrial protein homeostasis. Biochimica et Biophysica Acta (BBA)-Molecular Cell Research, 2013. 1833(2): p. 388-399.

[51] Nolden, M., The m-AAA protease defective in hereditary spastic paraplegia controls ribosome assembly in mitochondria. Cell, 2005. 123(2): p. 277-289.

[52] Ishihara, N., Regulation of mitochondrial morphology through proteolytic cleavage of OPA1. The EMBO journal, 2006. 25(13): p. 2966-2977.

[53] Yang, Q.-H., Omi/HtrA2 catalytic cleavage of inhibitor of apoptosis (IAP) irreversibly inactivates IAPs and facilitates caspase activity in apoptosis. Genes \& development, 2003. 17(12): p. 1487-1496.

[54] Cilenti, L., Inactivation of Omi/HtrA2 protease leads to the deregulation of mitochondrial Mulan E3 ubiquitin ligase and increased mitophagy. Biochimica et Biophysica Acta (BBA)-Molecular Cell Research, 2014. 1843(7): p. 1295-1307.

[55] Jin, S.M., Mitochondrial membrane potential regulates PINK1 import and proteolytic destabilization by PARL. Journal of Cell Biology, 2010. 191(5): p. 933-942.

[56] Germain, D., Ubiquitin - dependent and - independent mitochondrial protein quality controls: implications in ageing and neurodegenerative diseases. Molecular microbiology, 2008. 70(6): p. 13341341.

[57] Bragoszewski, P., The ubiquitin-proteasome system regulates mitochondrial intermembrane space proteins. Molecular and cellular biology, 2013. 33(11): p. 2136-2148.

[58] Käser, M. and T. Langer. Protein degradation in mitochondria. in Seminars in cell \& developmental biology. 2000. Elsevier.

[59] Lecker, S.H., A.L. Goldberg, and W.E. Mitch, Protein degradation by the ubiquitin-proteasome pathway in normal and disease states. Journal of the American Society of Nephrology, 2006. 17(7): p. 1807-1819.

[60] Jeong, J.H., Effects of low dose quercetin: Cancer cell - specific inhibition of cell cycle progression. Journal of cellular biochemistry, 2009. 106(1): p. 73-82.

[61] Soubannier, V., A vesicular transport pathway shuttles cargo from mitochondria to lysosomes. Current Biology, 2012. 22(2): p. 135-141.

[62] Navarro-Yepes, J., Oxidative stress, redox signaling, and autophagy: cell death versus survival. Antioxidants \& redox signaling, 2014. 21(1): p. 66-85. 
[63] Horvath, S.E. and G. Daum, Lipids of mitochondria. Progress in lipid research, 2013. 52(4): p. 590-614.

[64] Sugiura, A., A new pathway for mitochondrial quality control: mitochondrial - derived vesicles. The EMBO journal, 2014. 33(19): p. 2142-2156.

[65] Schatz, G., The protein import system of mitochondria. Journal of Biological Chemistry, 1996. 271(50): p. 31763-31766. 